\title{
Improving the Resolution Obtained in Lensless Imaging with Spatially Shaped High-Order Harmonics
}

\author{
Daniel Treacher ${ }^{1}$, David T. Lloyd ${ }^{1}$, Kevin O'Keeffe ${ }^{2}$, Patrick N. Anderson ${ }^{1}$ and Simon M. Hooker ${ }^{1}$ \\ 1. Department of Physics, University of Oxford, Clarendon Laboratory, Parks Road, Oxford, \\ OX1 3PU, UK \\ 2. Department of Physics, Swansea University, Singleton Park, Swansea, SA2 8PP, UK
}

The resolution obtained with coherent diffractive imaging (CDI) is limited by a number of factors, one of which is the transverse coherence of the illuminating beam. For a successful reconstruction, it is accepted that the illuminating beam should have a lateral coherence length of at least twice the largest linear dimension of the sample [1].

Setups utilising an infrared (IR) laser driving high harmonic generation (HHG) in a noble gas producing radiation in the XUV range is a convenient combination of low cost, accessibility and a high, but not complete, degree of coherence [2]. This transverse coherence depends on the driver's transverse intensity profile and coherence [3]. Hence to increase the harmonic beam's coherence, one can increase the coherence of the IR driver and shape the driver such that, at focus, it exhibits a top-hat intensity profile, thereby reducing the deleterious impact of the intensity dependant phase on the harmonic coherence.

We used a standard CDI reconstruction code to find the resolution for varying values of $\xi$. $\xi$ is taken to be the ratio of the widths of the complex coherence factor (CCF) to the spot size of the (Gaussian) illuminating $\mathrm{x}$-ray beam. The resolution was taken to be the distance for the intensity of a reconstructed edge to increase from $10 \%$ to $90 \%$ of its peak value.
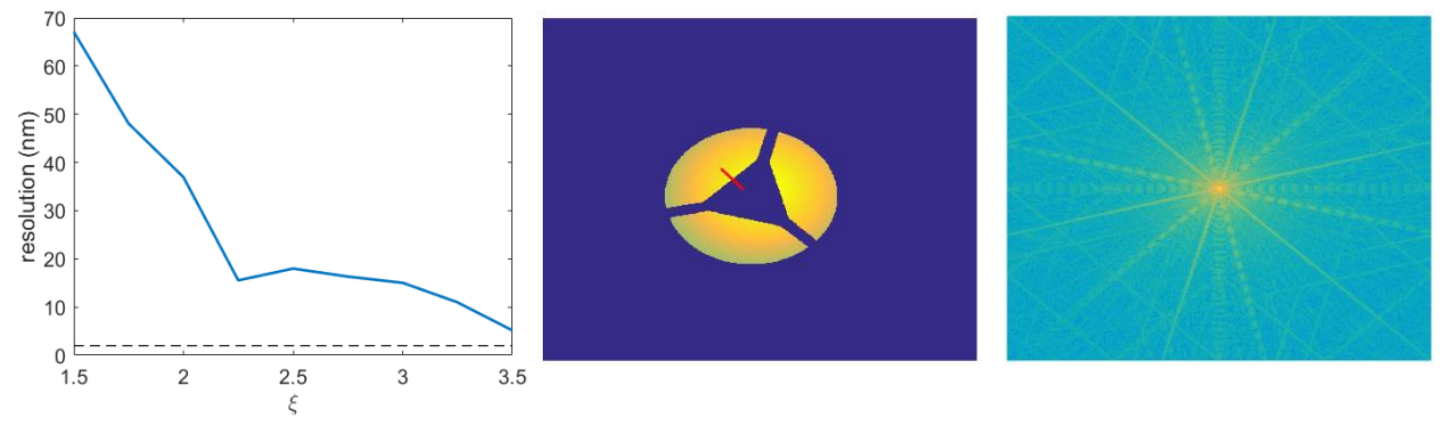

Fig. 1 Left: A plot of relative edge resolution against $\xi$ displaying the expected asymptotic behaviour toward the case of perfectly coherent illumination, denoted by the dashed line. Middle: The simple binary transmissive aperture used with an example 90/10 lineout section marked in red. The diameter of the outer circle is $7 \mu \mathrm{m}$. Right: Log plot of the diffraction pattern of the aperture.

In reference [4], the author's current laser system was found to have $\xi=1.5$, a figure supported by an auxiliary model that accounted for coherence contributions from the driver's transverse intensity and coherence. If one removes the intensity dependant contribution, $\xi$ was found to become approximately 3.5 . The plot above reveals that this improvement of roughly a factor of 2 in $\xi$ could increase the relative resolution by approximately a factor of 10 .

The shaping will be implemented experimentally with the use of a nematic 'liquid crystal on silicon' spatial light modulator (LCoS SLM) in reflection mode, placed upstream of the mirror focussing the shaped IR beam into the argon gas cell where HHG takes place.

The plot in figure 1 indicates that as $\xi$ is increased from the value currently applicable to the author's laser system, to the figure anticipated once the beam has been shaped, an order of magnitude increase in reconstruction resolution may be observed.

\section{References}

[1] J.C.H. Spence, U. Weierstall, M. Howells, "Coherence and sampling requirements for diffractive imaging" Ultramicroscopy, 101, 149 (2004)

[2] T. Ditmire, E. Gumbrell, R. Smith et al, "Spatial Coherence Measurement of Soft X-Ray Radiation Produced by High Order Harmonic Generation," Phys. Rev. Lett. 77, 4756 (1996).

[3] P. Salières, A. L'Huillier, M. Lewenstein, "Coherence control of high-order harmonics", Phys. Rev. Lett. 74, 3776 (1995).

[4] D. T. Lloyd, K, O'Keeffe, S.M. Hooker, "Complete spatial characterization of an optical wavefront using a variable-separation pinhole Pair", Opt. Lett. 38, 1173-1175 (2013) 